\title{
Reappraisal of Studies Concerning the Genetic Effects of the Radiation of Humans, Mice, and Drosophila
}

\author{
James V. Neel* \\ Department of Human Genetics, University of Michigan, Ann Arbor, Michigan
}

Key words: comparative radiation mutagenesis; species doubling doses; doubling dose of ioniz-

ing radiation; Drosophila; domestic mouse; humans; doubling dose of radiation

\section{INTRODUCTION}

Because of a number of recent developments, this seems a propitious time to reassess the relative sensitivities of humans, mice, and Drosophila to the genetic effects of ionizing radiation. As a population geneticist who is interested in the comparative sensitivity of various species to the genetic effects of ionizing radiation, I find these effects best expressed in terms of "doubling dose," defined as the amount of gonadal exposure to ionizing radiation that produces the same amount of mutation as occurs spontaneously each generation in the population under study. The concept is simple-deceptively simple, for, as Muller [1959] pointed out many years ago, there are numerous pitfalls in deriving this estimate and employing it in cross-species comparison. Ideally, as a population parameter it requires evaluating the sensitivity to radiation of the various germ cell stages throughout the prereproductive and reproductive portion of the organism's life cycle. Unfortunately, the chief alternative to deriving a doubling dose, namely, expressing effects simply as excess probability of mutations per locus per Gy for a chosen battery of genes radiated at a specific time in the life cycle, leaves one with little perspective in risk setting. Otherwise stated, the rate per locus in a specific sex at a specific point in the life cycle is an interesting laboratory parameter but the doubling dose is an absolutely basic parameter in evolutionary and population genetics, not to mention risk setting, which, not surprisingly, is much more difficult to evaluate. Although in most experimental studies the results are expressed in terms of gametic doubling dose, in the studies in Japan we are concerned with the results of exposure of one or both parents, and risk is best expressed in terms of zygotic doubling dose.

\section{HUMAN DATA}

The data most appropriate for attempting to estimate human sensitivities stem from the extensive studies in Hiroshima and Nagasaki, the first steps toward which were initiated in 1946, with continuing activity down to the present time. The findings of this major collaborative effort between U.S. and Japanese scientists have been summarized on a number of different occasions [cf. esp. Neel and Schull, 1991; Neel et al., 1997, in press] and I propose to be extremely brief. Over the years, a cohort of 31,150 children born to parents receiving significant amounts of radiation (i.e., within $2 \mathrm{~km}$ of the bomb's hypocenter) and a somewhat larger control cohort $(41,066)$ have been studied with respect to a variety of indicators: first, in the early years, for congenital defect, sex of child, physical development, and survival, then, in the middle years, for cytogenetic abnormality and, more recently, for the occurrence of malignant disease and for electrophoretic or functional defects in a battery of some 30 serum proteins or erythrocyte enzymes. None of these indicators was significantly related to parental radiation exposure but the net regression was slightly positive, but still nonsignificant. On the assumption that this small regression term was a radiation effect, and that we could estimate the contribution that spontaneous mutation makes each generation to the previously mentioned indicators, our estimate of the zygotic doubling dose was in the neighborhood of $2 \mathrm{~Sv}$ equivalents. There is a large but indeterminate error attached to this estimate, but we suggested the lower (95\%) boundary might be $1 \mathrm{~Sv}$ equivalent, whereas the upper boundary is indeterminate. We believe that in reaching this estimate our genetic assumptions regarding the impact of mutation in the preceding generation were quite conservative; in addition, we are keenly aware that the somewhat lower socioeconomic status of the exposed parents in the decade following the war could have contributed to the frequency of early death in their children [Kato et al., 1966], and so biased down-

Contract grant sponsor: National Institutes of Health; Contract grant number: CA26803.

Presented April 21, 1997, at the Symposium on Germ Cell Effects at the annual meeting of the Environmental Mutagen Society in Minneapolis, $\mathrm{MN}$.

*Correspondence to: James V. Neel, MD, PhD, Department of Human Genetics, Box 0618, M4708 Medical Science II, University of Michigan, Ann Arbor, MI 48109-0618.

Received 5 May 1997; revised and accepted 7 June 1997. 
ward the estimate of the doubling dose. If as much as half of the excess early deaths in the children of survivors were due to socioeconomic factors, this fact would increase the estimate of the doubling dose by roughly $100 \%$. It is extremely important to understand that the results are based on the radiation of a total population at all ages, rather than the usual experimental procedure of radiating a single sex at a convenient age.

A recent publication by Dubrova et al. [1996] would at first glance seem to present a serious challenge to this view of human radiosensitivity. I find that report to be seriously flawed and suggest the authors need either to verify their finding under a proper research design or retract the report. These authors claim that in children born in 1994, mutations in DNA minisatellites were twice as high in Belarus in children born to parents living in this area and exposed to fallout from the Chernobyl disaster than in control children. Unfortunately, the controls were drawn from England, a violation of the first principle in a study of this nature. Furthermore, the result is contradicted by an independent study on children born to atomic bomb survivors [Kodaira et al., 1995] and by preliminary studies of children born to parents of Chernobyl cleanup workers (H. Mohrenweiser, unpublished). In addition, the parental gonad doses resulting from the fallout in the Dubrova study were probably under .05 Sv equivalents of chronic radiation whereas in experimental studies on mice, the doubling dose of acute radiation for minisatellite mutations in spermatogonia was approximately $2.6 \mathrm{~Gy}$ [Dubrova et al., 1993; Sadamoto et al., 1994]. There is, thus, with allowance for the chronicity factor, at least a 100-fold discrepancy between the experimental mouse data and the findings reported in the Belarus study. Finally, these results suggest inherently implausible greater sensitivities to chronic radiation than the body of human data just reviewed or the mouse data we will consider next, both of these latter involving the more effective acute radiation. I'm sure you are all aware of the cloud of angst under which those exposed to the fallout from the Chernobyl are living; this report can only make an unnecessary contribution to a very sad situation.

\section{DOMESTIC MOUSE DATA}

Several years ago, after the summarization of the human data just presented, Susan Lewis and I undertook a detailed comparison of those data with the accumulated mouse data. Unfortunately, for reasons discussed in some detail elsewhere [Neel and Lewis, 1990], most notably the immaturity of the mouse fetus at birth and the intralitter competition effect both before and after birth, although effects of paternal radiation on the frequency of congenital defects, stillbirths, and early survival were demonstrated in the offspring of radiated male mice, much of the data cannot be compared with the human data. The most appropriate data for comparison with the human data would seem to be the results of the various specific locus-phenotype test systems, by far the most influential of which has been the 7-locus test system of Russell [1951]. So many important insights have issued from this system-e.g., the recovery of induced mutations from radiated female mice only in the first several post-radiation litters, the lesser genetic effectiveness of chronic as contrasted to acute radiation - that the system warrants special scrutiny. In his first report, Russell [1951] reported an average induced rate of $2.6 \times 10^{-7}$ per locus per $0.01 \mathrm{~Gy}$ for spermatogonia, and a spontaneous rate of $7.6 \times 10^{-6}$ per locus, concluding, after a review of the male Drosophila data then available (based on radiation of a mixture of male germ cell stages), that the mouse per locus rate was "considerably higher than that found in Drosophila.' (This statement was not in the context of a doubling dose.) On the basis of this and further data (see Table I), the estimate of the murine gametic doubling dose derived from the Russell data commonly employed in risk discussions has become $0.4-0.5$ Gy. The only other comparable mouse-specific multiplelocus data are those of Lyon and Morris [1966, 1969] who, on the basis of six different mouse loci, observed an induced rate in male spermatogonia following acute, relatively high-dose radiation of $7.6 \times 10^{-8} /$ locus $/ 0.01$ Gy, approximately one-third of the rate in the Russell experiments. Unfortunately, in their relatively small series no mutations were observed in the controls, so that no doubling dose can be computed from their data.

The results from eight different attempts to develop data from which a radiation doubling dose for mice could be calculated, based on more or less specific locus (or specific phenotype) approaches, are shown in Table I. These data are all male-based. Note the wide range in the various estimates, to which we found it impossible to attach errors in the usual statistical sense. Not shown there (because the data do not lend themselves to the calculation of a doubling dose) are the important results of Roderick [1983], who estimated for mice a per locus recessive lethal mutation rate in post-spermatogonial cells per locus from ionizing radiation of only $0.35 \times 10^{-8} / 0.01 \mathrm{~Gy}$, whereas for the Russell 7-locus system, the corresponding rate for all post-spermatogonial mutations was $45.32 \times$ $10^{-8} / 0.01 \mathrm{~Gy}$, approximately $80 \%$ of these mutations being homozygous lethal. As Roderick pointed out, this was about a 100-fold difference, in the direction of lesser sensitivity, although the error term to be attached to his estimate was large but difficult to calculate. The simple average of all the estimates in Table I, unweighted because we could think of no good way to weight the individual studies, was a male gametic doubling dose of acute radiation of $1.35 \mathrm{~Gy}$, with an indeterminate error.

There are several reasons to approach this estimate with caution. First, the data from many of the systems used in Table I are absolutely minimal for the generation of a doubling dose. The Oak Ridge data, which yield one of 
TABLE I. Summary of the Gametic Doubling Doses for Acute, "High-Dose'" Radiation of Spermatogonia*

\begin{tabular}{|c|c|c|c|}
\hline System & Data summarized in & $\begin{array}{l}\text { Doubling dose } \\
\text { (Gy) }\end{array}$ & $\begin{array}{c}\text { Origin of treated } \\
\text { males }\end{array}$ \\
\hline 1. Russell 7-locus & Ehling et al., 1985; Searle, 1974 & .44 & $101 \times \mathrm{C} 3 \mathrm{H}$ \\
\hline 2. Dominant visibles & Lüning and Searle, 1971 & .16 & various \\
\hline 3. Dominant cataract & Favor, 1989 & 1.57 & 101/E1 × C3H/E1 \\
\hline 4. Skeletal malformations & Ehling, 1966 & .26 & 101 \\
\hline 5. Histocompatibility loci & Bailey and Kohn, 1965 & $>2.60$ & C57BL/6JN \\
\hline \multirow[t]{3}{*}{ 6. Recessive lethals (3 studies) } & Sheridan and Wårdell, 1968 & $.51)$ & DBA \\
\hline & Lyon, 1959; Lyon et al., 1964 & $.80\} 1.77$ & $\mathrm{C} 3 \mathrm{H} / \mathrm{HeH} \times 101 / \mathrm{H}$ \\
\hline & Lyon, 1959; Searle, 1964 & 4.00 & $\mathrm{CBA}, \mathrm{C} 3 \mathrm{H}$ \\
\hline 7. Loci encoding for proteins & Neel and Lewis, 1990 & .11 & various \\
\hline \multirow[t]{2}{*}{ 8. Recessive visibles } & Lyon et al., 1964 & 3.89 & $\mathrm{C} 3 \mathrm{H} / \mathrm{HeH} \times 101 / \mathrm{H}$ \\
\hline & & Av. 1.35 & \\
\hline
\end{tabular}

*Yielded by the various specific-locus/specific-phenotype systems developed in the laboratory mouse, after Neel and Lewis [1990]. References to the sources of the data and the doubling dose calculations will be found in Neel and Lewis [1990].

the lower doubling dose estimates, should dominate the estimate, forcing us to look at these data with great care. Second, in his very first papers Russell recognized that the assumption that the loci he studied were representative of the genome was key. There are now data for the mouse indicating a 7-fold range in the rate per locus with which spontaneous mutation results in phenotypic effects [Green et al., 1965; Schlager and Dickie, 1967]. In Russell's data, radiation produced 18 times more mutations at the $s$ locus than at the $a$ locus, surely a signal to extrapolate with caution [reviewed in Searle, 1974].

Third, the mouse doubling-dose estimates of Table I are male-based. The demonstration [Russell, 1965] that although in the first few litters post-treatment the offspring of radiated female mice exhibited about the same amount of genetic damage as the offspring of radiated male, but that there was no apparent damage in the later litters of these same females, created a dilemma for risk setting. Was the human female similar to the mouse female in this respect? To be conservative, in extrapolating to the human situation, the mouse male-derived risks have usually been applied to both sexes. The zygotic doubling dose based on the data of Table I would thus become 2.7 Gy, but because of the lack of induced mutations in the late litters of females subjected to ionizing radiation this is almost certainly an underestimate of the mouse zygotic doubling dose. In the Japanese data, by contrast, radiated females contribute about half the dose on which the doubling dose estimate is based.

The fourth reason why the murine-based estimate of 1.35 Gy may be conservative is the apparent failure in the past to include either the observed cluster mutations or the mosaic mutations encountered in the studies of the Russells in the doubling dose estimates derived from their data by various groups. With respect to cluster mutations, over 30 years ago L.B. Russell [1964] described some 40 specific locus mutations which in the course of the experiment at Oak Ridge occurred in the offspring of both irradiated and control mice as clusters of two or more.
Of these, " 21 had one irradiated parent and 19 came from a contemporary control population of slightly smaller size." It is not clear how many of these occurred in the basic 7-locus series that provided the mutation rates quoted above. Selby [1996] in a brief abstract has recently suggested that because of the failure to incorporate clusters into the calculations, "the size of the doubling dose has been underestimated by at least a factor of three." These clusters, apparently reflecting a relatively high mutation rate in the "perigametic - very early zygote" interval [see Muller, 1959], are well documented in humans and Drosophila and have been, by purpose or default, included in past doubling dose estimates for these species [reviewed in Woodruff and Thompson, 1992]. The Drosophila data, however, suggest that only some $40 \%$ of all spontaneous mutations occur as clusters, so that while their omission from a calculation of the doubling dose for Drosophila would have biased the estimate downward, it would not be by a factor of three.

With respect to mutations first detected as mosaics, recently Russell and Russell [1996] have described a series of some 37 mosaic mutants that over the years have appeared in the F1 of both radiated and control mice, none of which have apparently been incorporated into the doubling dose calculations of the past that utilized the Russell data. These mutations occur in both males and females and are classified as "visible" or "masked." For technical reasons, they argue that most such mosaics "result from a single strand spontaneous mutation subsequent to the last premeiotic mitosis and before the first postmeiotic one of a parental genome- the "perigametic interval',' and calculate that the inclusion of this type of spontaneous mutation in the background rate would increase the spontaneous rate some 2.2 times over that calculated from singletons alone. Thus, this additional source of spontaneous mutation alone would, in the framework of a doubling dose, increase the earlier quoted estimate of the doubling dose for this system by a factor of approximately 2 . 
As a population geneticist working with a non-experimental organism, I do not enjoy the luxury of manipulating my material as does the experimentalist, a luxury that can, however, lead to over-simplification of a complex issue. From the population standpoint, there are both theoretical and practical reasons why cluster and mosaic mutations must be properly incorporated into the doublingdose issue. First, when Mother Nature goes to work on a newly fertilized egg carrying a mutant gene not present in either parent, she (or, more technically, the process of natural selection) does not ask exactly when and how that mutation originated. She evaluates the totality of all the newly arisen mutations represented in the zygote, which is what we have, in effect, attempted to emulate in the study in Japan. Second, in the past, clusters have certainly been included in the studies on radiation-induced sexlinked lethals in Drosophila. Meaningful comparisons between the large body of Drosophila data and the mouse data are impossible without including the clusters in the mouse data. The same is true in principle for comparisons involving the human data, where, because of the small sibship size, cluster detection is more difficult than for mice and Drosophila. Third, although the frequency of clusters may not be altered by radiation under the special circumstances of the design of the Russell study, with the radiation usually delivered at the 12th week of age, in the human experience, such as the exposures from the atomic bombs or the Chernobyl disaster, exposure to both sexes is at all ages and all stages of gametogenesis or fetal development, including the period particularly susceptible to the occurrence of what will become "clustered mutations." Until the full body of the Russell data are laid out in an appropriate fashion it is impossible to estimate the magnitude of the correction to the doubling dose estimate derived from the specific locus data, and we shall for now continue to employ the male gametic doubling dose estimate of $1.35 \mathrm{~Sv}$ equivalents developed by Neel and Lewis [1990] and derive the zygotic doubling dose by simply doubling the male estimate, knowing these estimates will almost surely be revised upward in the future.

\section{DROSOPHILA DATA}

With this apparent convergence of the doubling dose estimates for humans and mice, Drosophila was now the outlier; it was this discrepancy that prompted the reevaluation of the Drosophila experiments that I now present. It seemed a paradox, given the present understanding of the molecular basis of mutation and DNA repair, that an animal with the life strategy of Drosophila would be less sensitive per generation to the genetic effects of radiation than two mammals. Most of the Drosophila studies have been conducted at dose rates that, by the standards of human exposure, are ridiculously high. Furthermore, the experiments very predominantly involve the radiation of a mixture of germ cell stages, rather than the gonial stages, on which most of the mouse and human data are based. We will present where possible gonial data obtained at relatively low doses.

\section{Specific Locus Studies}

The first study on Drosophila melanogaster very specifically directed at mutation in spermatogonia (as well as mature sperm) resulting in visible recessive mutations is that of Alexander [1954], employing a dose of $30 \mathrm{~Gy}$ for the exposure of mature germ cells but 9 Gy for spermatogonia. The study was based on recessively-inherited visible mutations at eight third-chromosome loci. The average induced mutation rate for mature sperm was $6.0 \times$ $10^{-8} / 0.01 \mathrm{~Gy} /$ locus, and for spermatogonia, $1.5 \times 10^{-8} /$ $0.01 \mathrm{~Gy} /$ locus. This suggests a conversion factor of 4 in extrapolating from spermatozoal to spermatogonia doubling doses. The data are complicated by how the author elected to count clusters and incorporate them in her calculations. Unfortunately, no mutations were observed in 364,032 control locus tests, really most unusual, rendering a doubling dose estimate impossible. However, in six other multi-locus experiments [summary in United Nations, 1958, Table II], plus the data of Schalet [1960], the average spontaneous rate/locus for recessive visibles was $2.0 \times 10^{-5} /$ locus/generation. The use of this figure yields a doubling dose estimate for spermatogonia of $(2.0 \times$ $\left.10^{-5}\right) /\left(1.5 \times 10^{-8}\right)=13.3$ Gy for these loci. However, in addition to 71 proven mutants in the radiation series for mature sperm, there were 190 flies with the appearance of true mutations that were either sterile or died before the completion of breeding tests. (The corresponding number for the controls or spermatogonial series is not given.) Some correction for these seems indicated. If only a minority of these 190 , such as another 71 , were radiation-induced mutants, then the induced rate is $12.0 \times 10^{-8}$ Gy/locus, and the doubling dose is $6.7 \mathrm{~Gy}$. At this time point, a comparison of these findings with those of Russell [1956] did, indeed, suggest, as Russell had indicated, that, on the basis of the 7-locus system, the mouse was much more sensitive to the genetic effects of ionizing radiation than Drosophila - but there were more data to come.

A risk estimate for populations, as noted earlier, requires data on both sexes. Muller [1959], summarizing the work of himself and associates on visible mutations at some 8 loci, estimates the doubling dose for oogonia to be about $3.5 \mathrm{~Gy}$, and also estimates a factor of 2 greater sensitivity of oocytes than oogonia. Glass [1956; see also Glass and Ritterhof, 1956], in an experiment conducted at $3250 r$ with Drosophila females, involving 8 loci on chromosome 2, concluded: "If one may accept the average spontaneous mutation rate per locus estimated for 9 selected loci in the X-chromosome of oocytes by Muller et al. [1950] as being comparable to the average rate for the loci now studied in chromosome 2 , it follows that as 
little as $50 r$, but more likely around $200 r$, would double the spontaneous rate in oocytes.' The preference for the higher rate was due to the perception of an elevated control rate in Muller's data. Using the conversion factor of 2 derived from Muller's study, this would translate to a doubling dose for oogonia of about 4.0 Gy.

A somewhat neglected study has been that of Ives [1954], also conducted at $30 \mathrm{~Gy}$, who, employing essentially the same group of third chromosome loci as Alexander [1954], observed, following male radiation, an induced rate of $14 \times 10^{-8} /$ locus $/ 0.01 \mathrm{~Gy}$. Inasmuch as the rate is based on eggs laid through the 18th day after treatment and mating, the offspring result from the radiation of a mixture of germ cell stages. This rate is higher than either the spermatozoal or the spermatogonial rates reported by Alexander, i.e., indicated a greater radiation sensitivity than did Alexander's study. There were no controls in Ives' study, but employing the same controls as assembled above one arrives at a doubling dose estimate of $100 \times\left(2.0 \times 10^{-5}\right) /\left(14 \times 10^{-8}\right)=1.4 \mathrm{~Gy}$. To the extent this estimate is based on spermatocyte rates, it is too low, possibly by as much as a factor of 3-4. Ives [1954] drew attention to differences in how Russell and Alexander had scored apparent mutations that were infertile, and also how disproportionately a single locus, spotted, had contributed to the murine induced rate ( 25 of the 54 mutations) in Russell's [1951] original report, a fact Russell had also explicitly recognized. Ives concluded that the case for a significantly greater genetic sensitivity of the mouse than Drosophila to the genetic effects of ionizing radiation had not been made, a conclusion Russell [1956] challenged vigorously, principally because of Ives' failure to draw a clear distinction between the results of the radiation of mature sperm and of spermatogonia. It was in this discussion that Russell derived the estimate of 15 as the ratio of the mouse-induced rate to the Drosophila rate following acute spermatogonial radiation that has so frequently been used in discussions; this ratio is specific for the Russell mouse and Alexander Drosophila systems. The average of the spermatogonial-based estimates of Alexander (which I correct to $6.7 \mathrm{~Gy}$ ) and the mixed-stages study of Ives (corrected to $5.6 \mathrm{~Gy}$ ) is 6.2 Gy. Averaging this with the female rates of 4.0 Gy derived by Glass and 3.5 obtained by Muller, to obtain an estimate for a mixed-sex population, yields a figure of 5.0 Gy.

\section{Dominant Minute Test}

Glass [1955] studied the production of dominant Minute mutations at the relatively low dose of $10 \mathrm{~Gy}$ (as well as at other higher doses). The spontaneous rate was $8 \times 10^{-4} /$ fly/generation. At $10 \mathrm{~Gy}$, there was no difference between male and female rates, the total rate being $5 \times$ $10^{-3}$, or $5 \times 10^{-6} / 0.01 \mathrm{~Gy} / \mathrm{fly}$. The doubling dose is $(8 \times$ $\left.10^{-4}\right) /\left(5 \times 10^{-6}\right)$, or $1.3 \mathrm{~Gy}$. No correction for a difference between the sexes is necessary, but allowance for a factor of 4 difference between the rates in mature and immature gametes (see above) would increase this estimate to 5.2 Gy for Minute mutations.

\section{Sex-Linked Lethal Test}

The test for the induction of sex-linked lethals has been the most widely employed of all the Drosophila test systems, being the test with which Muller [1927] first demonstrated the mutagenic effect of X-rays in animals. Some of the early data based on mature germ cells suggested a male gametic doubling dose as low as 0.4 Gy [TimoféeffRessovsky, 1934; quoted in Spencer and Stern, 1948]. Fortunately, for our purpose, Spencer and Stern [1948] have carried the observations down to a dose of $0.25 \mathrm{~Gy}$. For all observations on the results of radiating males up to a dose of $10 \mathrm{~Gy}$, the control rate was $0.104 \%$, whereas the induced rate per 0.01 Gy was $0.002 \%$, resulting in a doubling dose estimate of $0.5 \mathrm{~Gy}$. There are no specific data on the sensitivity of immature gametes, but the circumstances of the study suggest the data are predominantly based on the radiation of mature sperm cells. Extrapolating from Alexander's study concerning the sensitivity difference between mature and immature germ cells, using a factor of 4 as before because the radiation involved mature germ cells, their doubling dose for sexlinked lethals in males becomes $0.5 \mathrm{~Gy} \times 4=2.0 \mathrm{~Gy}$.

Muller [1959], again summarizing the work of himself and others, has estimated that the doubling dose for recessive sex-linked lethals in Drosophila oogonia is about 3.5 Gy. For spermatogonia, the estimate was $10.0 \mathrm{~Gy}$, but with a wide error. Abrahamson and associates have also conducted extensive experiments on the induction by acute ionizing radiation of sex-linked lethals in Drosophila spermatogonia and oogonia, over a wide range of exposures. For spermatogonia at $30 \mathrm{~Gy}$, the observed doubling dose was approximately 7.4 Gy [Abrahamson and Friedman, 1964]; for oogonia, approximately 5 Gy [Abrahamson et al., 1981]. The average for these three malebased estimates is $6.5 \mathrm{~Gy}$ and for the two female-based estimates, $4.3 \mathrm{~Gy}$, with the average across sexes $5.4 \mathrm{~Gy}$. As Muller [1959] pointed out, these estimates may be biased upward by the induction of multiple mutations (scored as one) in a single lethal $\mathrm{X}$ chromosome, especially at the high radiation doses employed.

Furthermore, from a consideration of the sex-linked visible mutations incidentally observed in their lethaloriented experiments, Spencer and Stern [1948] wrote: ". . . the tentative conclusion was reached that the same $\mathrm{X}$-ray dosage/mutation rate relation holds for sex-linked visibles as for sex-linked lethals" (p. 69). Thus, their estimate of the male gonial doubling dose for visible mutations, derived as above for lethals, becomes $2.0 \mathrm{~Gy}$. The simple average of this estimate for males with that derived from an evaluation of Alexander's data (6.7) and the Ives' data (5.6) is 4.8 Gy. Combining this estimate with that 
of Muller (3.5) and of Glass (4.0 Gy) for females, to yield an estimate for a breeding population, results in an estimate of $4.3 \mathrm{~Gy}$ as the doubling dose for visibles.

\section{Combined Estimate}

The average of these three estimates of a gamete population doubling dose for the radiation of mainly immature Drosophila germ cell stages is [4.3 (visibles) + 5.0 (Minutes) +5.4 (lethals) $] / 3=4.9$ Gy. The corresponding estimate of the zygotic doubling dose would be $9.8 \mathrm{~Gy}$.

\section{PERSPECTIVE}

The estimates we have derived of the zygotic doubling dose of acute ionizing radiation for humans, mice, and Drosophila melanogaster are 2.0 Sv equivalents, $2.7 \mathrm{~Gy}$, and 10.0 Gy, respectively. Many assumptions have entered into these estimates; the errors are large, and there are reasons to suspect the estimates for humans and mice are underestimates. Those who disagree are invited to tender their own estimates. To me, the relative agreement between species with such different life histories is striking. The next step in a comparison such as this would usually be an extrapolation to the genetic effects of lowlevel, "chronic" radiation. Here, too, many debatable assumptions and extrapolations would be necessary; for present purposes, it is not necessary to enter into that contentious territory.

It has for some time been apparent that despite the enormous differences in duration of life, number of germ line cell divisions, mean body temperature, and reproductive strategies, the spontaneous mutation rates in Drosophila, mouse, and humans are "surprisingly" similar [reviewed in Neel, 1983; see also Drost and Lee, 1995]. The frequent occurrence of mutator events in Drosophila complicate the comparison (but they may also occur in mice and humans). Now it appears that the amount of ionizing radiation necessary to double that spontaneous rate may also agree within a factor of approximately 4 or even less. These comparisons are still imprecise and soft but, if in general correct, will certainly call for many discussions in the future concerning evolutionary strategy. Have higher eukaryotes through differences in DNA repair systems or the efficiencies of these systems "adjusted" to respond per generation to a mutagenic insult in proportion to their spontaneous mutation rate per generation? Or is this to some extent a matter of gene or nuclear target size [cf. Abrahamson et al., 1973] (but recall how much of the human genome is thought to be "junk", DNA)? The Drosophila spontaneous rate per unit time would of course be much greater than the mouse rate, and this in turn much greater than the human rate, but how and why, then, the "adjustment'" (if this is not coincidence) that results in the same relative genetic response to ionizing radiation per generation?
With the advent of technologies for the direct study of DNA for spontaneous and induced mutational events, the ability to cut through the phenotypic vagaries that have made cross-species comparisons so difficult is at hand. It is now possible to examine directly the same type of (DNA) indicator in all three species. With respect to human studies, almost 10 years ago the staff of the Radiation Effects Research Foundation (RERF) in Hiroshima began the major task of establishing a series of Epstein-Barr virus-transformed lymphocytoid cell lines from mother/ father/child trios, for half of which one or both parents had been exposed to the radiation of the A-bomb, the other half involving no such exposures. The ultimate goal is 600 in each series.

Multiple ways of visualizing genetic variation in DNA in these cell lines are now being explored [reviewed in Neel, 1995]. In particular, recent technical developments permit the two-dimensional display of an enzymatic digest of human genomic DNA in which the fragments have been isotopically labeled. We have now validated the use of these gels for genetic studies [Asakawa et al., 1994, 1995; Kuick et al., 1995, 1996], especially with regard to insertion/inversion/deletion events. With the development of this system, the ability to pursue absolutely comparable studies of the genetic effects of radiation in species as diverse as Drosophila melanogaster, the domestic mouse, and humankind is now at hand. Pilot studies on the efficiency of this and several other DNA-based techniques are now underway at RERF and in our Michigan laboratory, based on both a mouse model and the children of atomic bomb survivors - for the latter employing the cell lines described above. These studies have the potential not only to provide a firmer estimate of human risks but also to clarify the extent of the differences between mice and humans with respect to the genetic effects of radiation. Whether the resources will be there to undertake this long and arduous comparison is another question.

\section{ACKNOWLEDGMENTS}

The author thanks Drs. James Crow, Seymour Abrahamson, Ron Woodruff, and Susan Lewis for their helpful comments.

\section{REFERENCES}

Abrahamson S, Bender MA, Conger AD, Wolff S (1973): Uniformity of radiation-induced mutation rates among different species. Nature 245:460-462.

Abrahamson S, Friedman LD (1964): X-ray induced mutations in spermatogonial cells of Drosophila and their dose-frequency relationship. Genetics 49:357-361.

Abrahamson S, Meyer HU, DeJongh C (1981): The shapes of the radiation dose-mutation response curves in Drosophila: Mechanisms and implications. In Berg GG, Maillie HD (eds): “'Measurement of Risks.' New York: Plenum Publishing, pp 477-496.

Alexander ML (1954): Mutation rates at specific autosomal loci in the 
mature and immature germ cells of Drosophila melanogaster. Genetics 39:409-428.

Asakawa J, Kuick R, Neel JV, Kodaira M, Satoh C, Hanash SM (1994): Genetic variation detected by quantitative analysis of end-labeled genomic DNA fragments. Proc Natl Acad Sci USA 91:90529056.

Asakawa J, Kuick R, Neel JV, Kodaira M, Satoh C, Hanash SM (1995): Quantitative and qualitative genetic variation in two-dimensional DNA gels of human lymphocytoid cell lines. Electrophoresis 16:241-252.

Drost JB, Lee WR (1995): Biological basis of germline mutation: Comparisons of spontaneous germline mutation rates among Drosophila, mouse, and human. Environ Mol Mutagen 25 (Suppl. 26): $48-64$.

Dubrova YE, Jeffreys AJ, Malashenko AM (1993): Mouse minisatellite mutations induced by ionizing radiation. Nature Genet 5:92-94.

Dubrova YE, Nesterov VN, Krouchinsky NG, Ostapenko VA, Neumann R, Neil DL, Jeffreys AJ (1996): Human minisatellite mutation rate after the Chernobyl accident. Nature 380:683-686.

Glass B (1955): A comparative study of induced mutation in the oocyte and spermatozoa of Drosophila melanogaster. II. Deficiencies and minutes. Genetica 40:281-296.

Glass B (1956): Differences in mutability using different stages of gametogenesis in Drosophila. Brookhaven Symposia Biol 8:148170 .

Glass HB, Ritterhof RK (1956): Spontaneous mutation rates at specific loci in Drosophila males and females. Science 124:314-315.

Green EL, Schlager G, Dickie MM (1965): Natural mutation rates in the house mouse: Plan of study and preliminary estimates. Mutat Res 2:457-465.

Ives PT (1954): Radiation-induced mutation rates in Drosophila and mice. Am Nat 88:361-364.

Kato H, Schull WJ, Neel JV (1966): A cohort-type study of survival in the children of parents exposed to atomic bombings. Am J Hum Genet 18:339-373.

Kodaira M, Satoh C, Hiyama K, Toyama K (1995): Lack of effects of atomic bomb radiation on genetic instability of tandem-repetitive elements in human germ cells. Am J Hum Genet 57:1275-1283.

Kuick R, Asakawa J, Neel JV, Satoh C, Hanash SM (1995): High yield of restriction fragment length polymorphisms in two-dimensional separations of human genomic DNA. Genomics 25:345-353.

Kuick R, Asakawa J, Neel JV, Kodaira M, Satoh C, Thoraval D, Gonzalez IL, Hanash SM (1996): Studies of the inheritance of human ribosomal DNA variants detected in two-dimensional separations of genomic restriction fragments. Genetics 144:307-316.

Lyon MF, Morris T (1966): Mutation rates at a new set of specific loci in the mouse. Genet Res 7:12-17.

Lyon MF, Morris T (1969): Gene and chromosome mutation after large fractionated or infractionated radiation doses to mouse spermatogonia. Mutat Res 8:191-198.

Muller HJ (1927): Artificial transmutation of the gene. Science 66:8487.

Muller HJ (1959): Advances in radiation mutagenesis through studies on Drosophila. In Bugher JC (ed): "Progress in Nuclear Energy Series VI."' New York: Pergamon Press, pp 146-160.

Muller HJ, Valencia JI, Valencia RM (1950): The frequency of spontaneous mutations at individual loci in Drosophila. Genetics $35: 125$.
Neel JV (1983): Frequency of spontaneous and induced point mutations in higher eukaryotes. J Hered 74:2-15.

Neel JV (1995): New approaches to evaluating the genetic effects of the atomic bombs (Invited Editorial). Am J Hum Genet 57:12631266.

Neel JV, Lewis SE (1990): The comparative radiation genetics of humans and mice. Annu Rev Genet 24:327-362.

Neel JV, Schull WJ (1991): “The Children of Atomic Bomb Survivors: A Genetic Study." Washington, DC: National Academy Press.

Neel JV, Schull WJ, Awa AA, Satoh C, Kato H, Otake M, Yoshimoto Y (1990): The children of parents exposed to atomic bombs: Estimates of the genetic doubling dose of radiation for humans. Am J Hum Genet 46:1053-1072.

Neel JV, Asakawa J, Kuick R, Hanash SH (in press): Studies on the genetic effects of the atomic bombs: Past, present, and future. In Peterson L (ed): "Effects of Ionizing Radiation: Atomic Bomb Survivors and Their Children."

Roderick TH (1983): Using inversions to detect and study recessive lethals and detrimentals in mice. In de Serres FJ, Sheridan W (eds): "Utilization of Mammalian Specific Locus Studies in Hazard Evaluation and Estimation of Genetic Risk." New York: Plenum, pp 135-167.

Russell LB (1964): Genetic and functional mosaicism in the mouse. In Locke M (ed): "The Role of Chromosomes in Development." New York: Academic Press, pp 153-181.

Russell LB, Russell WL (1996): Spontaneous mutations recovered as mosaics in the mouse specific-locus test. Proc Natl Acad Sci USA 93:13072-13077.

Russell WL (1951): X-ray induced mutations in mice. Cold Spring Harb Symp Quant Biol 16:327-336.

Russell WL (1956): Comparison of X-ray-induced mutation rates in Drosophila and mice. Am Nat 90:67-80.

Russell WL (1965): Effect of the interval between irradiation and conception on mutation frequency in female mice. Proc Natl Acad Sci USA 54:1552-1557.

Sadamoto S, Suzuki S, Kamiya K, Kominami R, Doh K, Niwa O (1994): Radiation induction of germline mutation at a hypervariable mouse minisatellite locus. Int J Radiat Biol 65:549-557.

Schlager G, Dickie MM (1967): Spontaneous mutations and mutation rates in the house mouse. Genetics 57:319-330.

Searle AG (1974): Mutation induction in mice. In Lett JT, Adler HI, Zelle M (eds): “Advances in Radiation Biology." New York: Academic Press, pp 131-207.

Selby PB (1996): The doubling dose for radiation, or for any other mutagen, is actually several times larger than has been previously thought if it is based on specific-locus mutation frequencies in mice. Environ Mol Mutagen 27 (Suppl 27):61.

Spencer WP, Stern C (1948): Experiments to test the validity of the linear $\mathrm{r}$-dose/mutation frequency relation in Drosophila at low dosage. Genetics 33:43-74.

Timoféeff-Ressovsky NW (1934): "Experimentelle Mutations forschung in der Vererbungslehre." Dresden, Leipzig: Theodor Steinkopff.

Woodruff RC, Thompson JN (1992): Have premeiotic clusters of mutation been overlooked in evolutionary theory? J Evol Biol 5:457464.

Accepted byE. Zeiger 